\title{
A functionality, safety and validity study of innovative REACH devices
}

\author{
Dominika Kozak $\mathrm{PhD}^{\mathrm{a}, *}$ \\ Simon Burgermeister $\mathrm{MD}^{\mathrm{b}, \mathrm{c}}$ \\ Jean De Buretel De Chassey MSc ${ }^{d}$ \\ Adrien Naef ${ }^{\mathrm{b}, \mathrm{c}}$ \\ Alexandre Maringue $\mathrm{BSc}^{\mathrm{d}}$ \\ Damien Dietrich $\mathrm{MD}^{\mathrm{b}, \mathrm{c}}$
}

\begin{abstract}
${ }^{a}$ Alreh Medical, Warsaw, Poland; beHealth and Telemedicine Division, Geneva University Hospitals, Switzerland; 'Radiology and Medical Informatics Department, University of Geneva, Faculty of Medicine, Switzerland; 'Department of Internal Medicine, Rehabilitation and Geriatrics, Geneva University Hospitals, Switzerland; *Corresponding author: dominika.kozak@alreh.pl
\end{abstract}

\begin{abstract}
D. Kozak, S. Burgermeister, J.D.B. de Chassey, A. Naef, A. Maringue, D. Dietrich. A functionality, safety and validity study of innovative REACH devices. Gerontechnology 2017;16(3):181-188; https://doi.org/10.4017/gt.2017.16.3.007.00 Introduction Ageing is one of the major challenges affecting health-care systems in developed countries. Sedentary lifestyle is common among elderlies and associated with increased mortality, morbidity and decreased autonomy and quality of life. Accordingly, one cornerstone of the REACH project is to promote physical activity. In particular, in touchpoint cluster 1, a personal mobility device integrating a monitoring solution will be created. This study aims to assess the safety, validity and functionality of devices that may be adapted for this purpose: a rehabilitation equipment produced by Alreh Medical, the iStander activ, its software, Neuroforma, and a sensor, the Fitbit Charge 2. Methods Patients hospitalized at the geriatric division of Geneva University Hospitals and healthy controls were recruited. Patients were randomly assigned to train their transfers with the iStander activ and its associated-software, neuroforma, or according to the Standard Medical Care during 4 consecutive days over 30 minutes. Healthy controls trained their transfers using the iStander activ and Neuroforma. Exercises were monitored by the Fitbit Charge 2. Safety was assessed by free reporting, functionality by the NASA Task-Load Index (NTLI) and by free commenting by patients and care-givers. Differences between heart rate values measured by the Fitbit device and those measured by care-givers assessed the accuracy of heart rate measurement by the Fitbit device. Results No major safety issues were reported. Functionality assessment by care-givers and patients concluded that the iStander in its current form is not properly suited for transfer training in this use case but would be more effective in situations requiring strong trunk stabilization such as upper limb exercises or lower limb exercises for patients suffering from severe lower-limb paresia. The verticalisation force was reported as too strong and the range of possible movements too limited. Some patients reported not being interested in associated videogame interface and suggestions of design improvements were made. The ease-of-use and comfort of the Fitbit were appreciated but the wristband was reported as difficult to adapt. The comparison of heart rate values raised concerns about the potential use of the Fitbit Charge 2 as a heart rate measurement tool for REACH. Conclusion: Our study, though exploratory, provides important insights for the development of the touchpoint cluster 1 personal mobility device.
\end{abstract}

Keywords: physical activity, elderly, functionality, rehabilitation, HR, mobility, senior

Ageing societies are now one of the biggest challenges of the modern world. Sedentary lifestyle, common in older adults, adversely affect a number of body functions, which are necessary to maintain the autonomy and independence in the daily functioning ${ }^{1}$.
Researches carried out in recent years have shown the connection between sedentary lifestyles and mortality. For example, Pitsavos et $\mathrm{al}^{2}$ examined 2172 patients with acute coronary syndromes. Active life before a coronary incident, assessed by the IPAQ (international physical activity questionnaire) questionnaire was associated 
with a lower hospital mortality and a lower likelihood of repeated cardiovascular events within the first 30 days after discharge from the hospital.

In addition to its positive effect on mortality, regular physical activity is one of the most important factors for the so-called positive ageing ${ }^{3}$. Physical activity plays an important role in better subjective perception of the quality of life among seniors. Numerous evidences show that regular physical activity is associated with better mobility, improved daily activities, as well as decreased anxiety and occurrence of depression in people over the age of $65^{4-6}$. The beneficial effect of exercises is particularly noticeable in older adults already affected by disabilities. Of note, systematic physical activity also positively affects autonomy ${ }^{6}$. Importantly, the use of simple forms of movement in such a way that physical activity is associated with pleasure, relaxation and fun is the most effective among seniors ${ }^{4}$.

As described in detail in this special issue of Gerontotechnology, the REACH project integrates the importance of active ageing to reduce the length of in-hospital stay and to promote the delivery of home care. In particular, in touchpoint cluster 1, a personal mobility device integrating a monitoring solution will be created.

In the Personal Mobility Device Cluster 1, activation programme is addressed to those older adults whose activity levels decreased for some reasons (including hospitalisation after one event), resulting in negative feedback loop: fear of physical activity leads to less activity which further increases the sense of fear of falling during an activity. The solution for this group of seniors is to create such an activating device that will provide the possibility of complementary motor training as well as activating mental processes at home.

To create such a device, an option would be to adapt existing solutions. As a first step towards this possibility, this exploratory study aims to gain the first insights about the safety, validity and functionality of a rehabilitation equipment prototype produced by Alreh Medical, the iStander, associated with its software, Neuroforma, as well as a commercially available sensor produced by Fitbit, the Fitbit Charge 2, in a set of patients matching the HUG use-case definition of the $\mathrm{REACH}$ project and in a set of healthy adults.

\section{Methods \\ Overview}

Patients hospitalized in the Geneva Geriatric Division and healthy adults matching our inclusion criteria were recruited. Patients were randomly assigned to train their transfers with the iStander and its associated-software, Neuroforma $(n=5)$, or according to the Standard Medical Care (SMC, $\mathrm{n}=5$ ) during 4 consecutive days over 30 minutes. Healthy adults $(n=5)$ trained their transfers using the iStander and Neuroforma. Exercises were monitored by the Fitbit Charge 2. Safety was assessed by free reporting of any adverse events by patients or care-givers. Functionality was assessed by the NASA Task-Load Index (NTLI) jointly filled in by patients and care-givers at day 4. Care-givers and patients were also invited to freely comment on the devices. Finally, a comparison of heart rate values measured by the Fitbit device and heart rate values measured by care-givers assessed the accuracy of the Fitbit Charge 2 heart rate measurement.

\section{Participants}

Patients to which physical therapy or occupational therapy was prescribed were screened for eligibility and eventually recruited by physical or occupational therapists. Healthy adults were recruited by the research team. The study was presented both orally and using a dedicated information form. After a minimal reflection period of 24 hours, informed consents were collected in a written form.

Inclusion criteria for patients were those of the REACH HUG use-case: age over 65 years old, and hospitalized at the Geneva geriatric Hospital (Hôpital des Trois Chênes), and planned discharged with the help of the Geneva Institution of home care (Institution Genevoise de Maintien à Domicile, IMAD), and a Mini-Mental State Examination (MMSE) $20<$ MMSE $<28$ or a Functionnal Independance Measure (FIM) below 6 in one or more of the scale items.

Inclusion criteria for healthy adults were: age between 30 and 80 years old and no active medical conditions interfering in any way with rehabilitation exercises. For example, a patient with hip pain due to osteoarthritis would not have been included in the study as the pain would have prevented him to use the equipment at its full range.

Moreover, both healthy adults and patients had a height comprised between $165 \mathrm{~cm}$ and $175 \mathrm{~cm}$ with a weight of no more than $90 \mathrm{~kg}$ in order to comply with the iStander active equipment available at HUG.

\section{Intervention}

Patients were randomly assigned to train their transfers with the iStander and its associatedsoftware, Neuroforma $(n=5)$, or according to the Standard Medical Care $(S M C, n=5)$ during 4 consecutive days over 30 minutes. Healthy adults $(n=5)$ trained their transfers using the iStander and Neuroforma. Exercises were monitored by the Fitbit Charge 2. 
The randomization sequence was 1:1. However, care-givers had the possibility to change a patient to another group in the case of logistical constraints (i.e: the Alreh equipment nonavailable). The next patient was then allocated to the second group. There was no allocation concealment and no blinding for care-givers, patients and the research team. This randomization procedure is obviously not ideal but matches the exploratory nature of our study.

Transfer refers to the action of moving from a position to another.Standard Medical Care refers to any procedures for transfer training at HUG. Exercises in this setting are adapted for every patient in order to best suit their needs. It may include the use of dedicated rehabilitation equipment. The possible usages of the iStander and Neuroforma software was demonstrated by the Alreh Medical team to hospital care-givers. They were then free to use it in a way that would best fit patients' needs for transfer training. The only constraint was the timing (4 consecutive days of training over 30 minutes).

\section{Endpoints}

The endpoints were the safety of the iStander and the Fitbit Charge 2, assessed by free reporting by patients and care-givers, the functionality of the iStander, its associated software and the standard medical care, assessed by the NASA Task-Load Index (NTLI), as well as the validity of the heart-rate measurement by the Fitbit Charge, assessed by the mean absolute difference in the heart rate measured by the Fitbit Charge and the heart rate measured by care-givers using the standard hospital equipment (Philips Mindray VS-900 and associated nellcor pulse oximeter).

\section{Devices}

The Fitbit charge 2 is a CE-labelled wrist wearable optical heart rate sensor that provides an estimation of the heart rate through detection of small arteries dilatation using a led directly in contact with the wrist skin. It also provides, nonexhaustively, an estimation of the oxygen consumption, energy expenditure, number of steps, quality of sleep, and walking distance. The psychometric properties of the Fitbit Charge were estimated in this study ${ }^{7}$.

The iStander is a CE-labelled rehabilitation device facilitating medium and long-term care at home for older adult users. iStander rehabilitation device is designed to activate the motor and mental activity of olderpeople whose daily activity levels decrease. The device is intended for individual use at home. iStander is used in the prevention of falls of older people and, due to the simultaneous combination of motor and mental exercises, effectively supports the cog- nitive processes. The device is equipped with a mechanism to assist standing up- GymUp, which allows for mobility exercises of ankle, knee and hip joints, and increases muscle strength of lower limbs by performing squats and back stances. A special corset and seat ensure safety during exercises to strengthen back and abdomen muscles by lifting legs. iStander also allows for independent, safe standing in the upright position, and safely performing balancing exercises as well as exercises that activate the upper part of a body using the multimedia programme (Figure 1).

The multimedia programme- Neuroforma, is also a part effectively supporting cognitive processes during physical and mental exercises. The associated software aims to empower training by managing the exercise routine and keeping the user motivated. It also includes brain exercises designed for older adults and neurological patients. Heart rate measurements by care-givers were performed with the standard HUG equipment (Philips Mindray VS-900 and associated nellcor pulse oxymeter). In the case of a bad signal quality with the pulse oxymeter, care-givers took manually the pulse on the radial artery, with a time watch.

\section{Procedures}

On Monday, the study was presented to candidate patients by care-givers and to healthy adults by the research team, both orally and via the information form. On Tuesday, participants' consents were collected via the informed consent form by care-givers. Patients were randomly assigned to train their transfers with the iStander or according to SMC. This training was performed under the supervision of occupational therapists and under monitoring by the Fitbit Charge 2. Training sessions lasted 30 minutes and were repeated every day until Friday (4 days total). Data from the sensor were collected daily in an anonymous manner. The starting time and ending time of the exercises as well as 3 measures
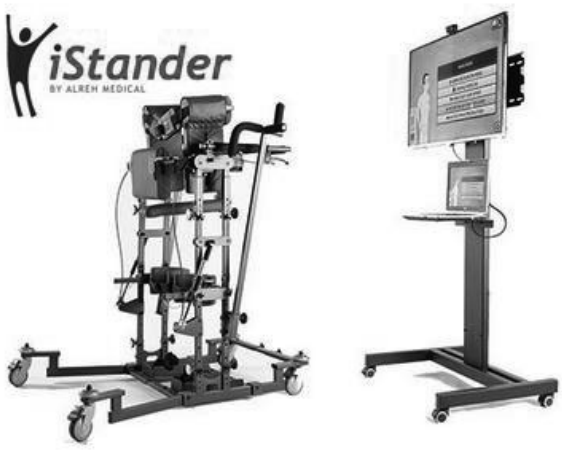

Figure 1. iStander device by Alreh Medical 
of heart rate (pre, post and during the exercise) were recorded daily and anonymously by caregivers using the Case Report Form (CRF). To guarantee anonymity as best as possible, caregivers allocated a number to patients on the CRF but stored no link between the patient's identity and the CRF (or Fitbit data). They paid attention not to give the same number twice. Healthy adults followed the same protocol but practiced only with the iStander only.

At the end of the week, the CRF including the NTLI questionnaire and the safety report were filled in by the occupational therapists and the patients in an anonymous manner. Basic medical and demographic data (age, sex, marital status, children, home help, diagnosis, treatments, MMSE score and functional independence measurement, activity of daily living evaluation) were collected by the care-givers in an anonymous manner. Moreover, patients and care-givers had the possibility to freely comment on the iStander and Fitbit Charge directly on the CRF. Adverse events were reported by patients and care-givers through the CRF. The occurrence of any serious adverse event would have led to the study interruption. These procedures are depicted in Supplementary Figure 1. The CRF, including the NTLI is attached as Supplementary Material 1.

\section{Data Management}

The NTLI questionnaire, the free functionality report, the safety report, the heart rate measurements and the demographic and medical information were filled in by the care-givers and the participants directly on the CRF. This was done in an anonymous manner with one number allocated to each participant and no code stored. Data collected by the sensors were copied by the care-givers to a dedicated, password-protected, hard drive and computer which also contained the filled-in CRFs. Data were labelled only with the participant number. With that protocol, the research team or any third party was prevented to access non-anonymized personal medical data. Data were stored on the hard drive and on a computer in our secure offices (campus biotech, Chemin des mines 9, 1202 Geneva) and further used for analysis.

This study was approved by the Geneva Canton Ethics Body (Commission Cantonale d'Ethique de la Recherche) under the number 2016-01957.

\section{Sample size}

Our sample size may appear as small. However, we believe it was adapted to our study main objective that was to identify critical problems in the safety, the validity or the functionality of innovative devices. A small sample size may induce the under recognition of devices functionality issues, notably because of patients' specific au- tonomy limitations. For example, if low effort tolerance is present for all our patients, functionality issues under more high-intensity training may be missed. The perfect solution would be to increase the sample size. However, at this stage of development in which we aim at identifying critical problems, we have decided to minimize this bias by including healthy adults that were able to use the iStander without any kind of autonomy limitation. For the same reason, healthy adults were not selected in an age-matched manner.

\section{Analysis}

The presence of safety issues reported for the iStander or the Fitbit Charge were asked to be reported immediately. Basic descriptive statistics on the pooled NTLI questionnaires were performed as well as comparison between the NTLI questionnaires between the three groups (healthy controls, patients SMC, patients iStander). Qualitative analysis of patients and caregivers inputs regarding functionality provided further insights. Finally, heart rate data collected by the Fitbit sensor were compared to heart rate data collected by care-givers using the mean absolute difference and standard deviation.

\section{Results}

\section{Comparison of the study groups}

Basic demographic and medical information of participants are depicted in Table 1. The mean age was 79.8 years in the SMC patient group and 89.6 years in the iStander patient group with a male to female ratio of $3 / 2$ and $4 / 1$ respectively. The total number of active pathologies highlights the importance of cardio-vascular diseases in both groups. Osteo-articular and endocrine diseases were also frequently encountered, with Osteo-articular diseases being overrepresented in the SMC patient group. The causes of hospitalization were diverse in both groups. As expected with our small sample size, the SMC patient group and the iStander patients group differed significantly in respect to several items. The healthy control group had a mean age of 42.4 years with a slight predominance of woman.

\section{Assessment of the heart rate measurement va- lidity by the Fitbit Charge 2}

During each exercise, simultaneous measures of HR by the Fitbit Charge and by care-givers were performed on 3 occasions, as described in the Methods Section. In the healthy adults group, all measures were successfully performed. In the SMC patients group and the iStander patients group, 48/60 and 45/60 measures were successfully performed, respectively. Reasons for missing data included loss of Fitbit data before transfer and omissions by care-givers. In total 153 absolute differences between the heart rate measured by the care-givers and the heart 
Table 1. Basic demographic and medical profile of participants (multiple pathologies are possible in one patient)

\begin{tabular}{|c|c|c|c|c|}
\hline & & $\begin{array}{l}\text { Healthy } \\
\text { controls }\end{array}$ & SMC patients & $\begin{array}{l}\text { iStander } \\
\text { patients }\end{array}$ \\
\hline & Age (mean, & $42.4(38,48)$ & $79.8(70,90)$ & $89.6(84,93)$ \\
\hline & Sex M/W & $2 / 3$ & $3 / 2$ & $4 / 1$ \\
\hline \multirow{11}{*}{$\begin{array}{c}\text { Total number of } \\
\text { Active pathologies } \\
\text { per category }\end{array}$} & Cardio- & 0 & 13 & 17 \\
\hline & Respiratory & 0 & 0 & 5 \\
\hline & Osteo-articular & 1 & 10 & 2 \\
\hline & Infectious & 0 & 3 & 2 \\
\hline & Gastro- & 0 & 1 & 3 \\
\hline & Endocrinologic & 0 & 5 & 3 \\
\hline & Uro-genital & 0 & 2 & 4 \\
\hline & Hemato- & 0 & 1 & 3 \\
\hline & Neurological & 0 & 1 & 3 \\
\hline & Immunological & 0 & 0 & 1 \\
\hline & Others & 0 & 0 & 2 \\
\hline \multirow{6}{*}{$\begin{array}{c}\text { Cause of } \\
\text { Hospitalisation per } \\
\text { category }\end{array}$} & Cardio- & 0 & 1 & 1 \\
\hline & Respiratory & 0 & 0 & 0 \\
\hline & Osteo-articular & 0 & 1 & 1 \\
\hline & Infectious & 0 & 1 & 2 \\
\hline & Gastro- & 0 & 0 & 1 \\
\hline & Others & 0 & 2 & 0 \\
\hline
\end{tabular}

sus 6) with a lower mental demand (4.8 versus 6$)$ at the cost of an increase physical demand (16.2 versus 14), effort (17.6 versus 15), frustration (6 versus 4.4) and temporal demand (12.4 versus 11.6). Unsurprisingly, the mental demand, physical demand, temporal demand and effort were lower in the healthy controls using the iStander compared to the patients using the iStander whereas the performance and the frustration were higher. rate measured by the Fitbit charge 2 were calculated. The mean absolute difference was 9.92 bpm with a standard deviation of 11.59 bpm. The mean difference was similar in the iStander patients and SMC patients group (8.73 and 9 bpm, respectively) but was slightly higher in the healthy control group (11.55bpm). In each group, important maximum differences were noted (around or above $50 \mathrm{mmHg}$ ). 30.7\% of all absolute differences were superior to $10 \mathrm{bpm}$. This percentage ranged from 35\% (healthy control) to $24.4 \%$ (iStander patients). Altogether, these results, summarized in Table 2, raise concerns about the validity of heart rate measurements by the Fitbit Charge 2 in our context and would warrant further investigations.

\section{Functionality assessment of the iStander and SMC by the NTLI scale}

Table 3 describes the results of the functionality assessment by the NTLI across the different study groups. For patients using the iStander, the NTLI indicated a low mental demand (4.8), a low frustration (6), a high physical demand (16.2) and effort (17.6) for a medium performance (7.2) and temporal demand (12.4). Compared to the SMC, the iStander seems thus to be slightly more efficient (7.2 ver-
High efforts and physical demand may be required during a rehabilitation process. It is thus difficult to draw conclusions for these items on the basis of the NTLI. Moreover, the comparison between the SMC and iStander patients group should not be over interpreted as it is based on small sample sizes with groups differing in various aspects. The functionality assessment by free reporting below is more helpful. However, our results using the NTLI illustrate that the global performance of the iStander should be improved and the frustration associated to its use, decreased.

\section{Functionality assessment of the iStander and the Neuroforma software by free reporting}

The ease of mounting and unmounting of the iStander was appreciated by care-givers that found it also well suited for squats but not for other inferior limbs exercises, in particular for transfer training. Care-givers also reported that although the iStander was adapted for upper limbs training by providing a useful trunk stabilization, the need for this type of exercise was rarer in the hospital setting. The verticalisation force was reported as too strong for our patients and should be adapted. Finally, they would have appreciated if the iStander could propose more functions than verticalisation. Overall, the conclusion of care-givers was that the iStander in its current form is mainly useful in situations in which the trunk needs to be stabilized to allow
Table 2. Absolut differences in heart rate measurements by Fitbits and caregivers. $n=$ number of measurements in each group. std = standard deviation and $\Delta=$ difference. All measurements are in beats per minute

\begin{tabular}{l|cccc}
\hline & $\begin{array}{c}\text { Healthy adults } \\
\mathrm{n}=60\end{array}$ & $\begin{array}{c}\text { SMC patients } \\
\mathrm{n}=48\end{array}$ & $\begin{array}{c}\text { iStander patients } \\
\mathrm{n}=45\end{array}$ & $\begin{array}{c}\text { Total } \\
\mathrm{n}=153\end{array}$ \\
\hline mean $|\Delta|($ std $)$ & $11.55(12.34)$ & $9(10.16)$ & $8.73(11.98)$ & $9.92(11.59)$ \\
\hline $\max |\Delta|$ & 48 & 59 & 54 & 59 \\
\hline$\%|\Delta|>10 \mathrm{bpm}$ & 35 & 31.3 & 24.4 & 30.7 \\
\hline
\end{tabular}


Table 3. Mean NTLI scores per item and group. Scale from 1 to 20 with standard deviation reported under parenthesis. SMC $=$ standard medical care

\begin{tabular}{lccc}
\hline & $\begin{array}{c}\text { Healthy } \\
\text { controls using } \\
\text { iStander }\end{array}$ & $\begin{array}{c}\text { Patients } \\
\text { following SMC }\end{array}$ & $\begin{array}{c}\text { Patients using } \\
\text { iStander }\end{array}$ \\
\hline Mental Demand & $4(3.3)$ & $6(3.4)$ & $4.8(1.5)$ \\
\hline Physical Demand & $6.2(5.5)$ & $14(2)$ & $16.2(3.1)$ \\
\hline Temporal Demand & $9.6(4.6)$ & $11.6(1.7)$ & $12.4(2.3)$ \\
\hline Performance & $8.6(6)$ & $6(1)$ & $7.2(6.8)$ \\
\hline Effort & $8.4(5.5)$ & $15(4.7)$ & $17.6(2.2)$ \\
\hline Frustration & $7.8(5.6)$ & $4.4(1.3)$ & $6(4.8)$ \\
\hline
\end{tabular}

proper training of upper-limbs or training of severely paretic lower limbs. It may be more suitable for an intense use in neurorehabilitation. As motivation is an important aspect of rehabilitation and return to active life, a modern multimedia platform- Neuroforma for independent motor and cognitive exercises includes sets of exercises which aims to makes them good fun. Watching the results is a good motivation to daily exercises.

Regarding Neuroforma, care-givers and users appreciated the clear interface and the ease ofuse but suggested an improvement of the design. Some crashes were reported in the program as well as a hyper-sensitivity to light variation in the room. Finally, some participants demonstrated only a low interest for video games.

The combination of mechanical device and associated software gives the opportunity to exercise and in the same time activate the cognitive functions while performing different tasks.

The used games require further improvements in such a way to engage a person deeper.

\section{Functionality assessment of the Fitbit Charge 2 by free reporting}

Care-givers and patients appreciated the ease and comfort of use. The use of Fitbit also promoted patient empowerement. However, the wrist-band was reported as difficult to adapt. A suggested improvement was the addition of an alert in case of high or irregular heart rates.

\section{Safety assessment by free reporting}

The only adverse event reported was the premature wear of one iStander wheels. This did not lead to any harm to participants. No serious adverse events were reported.

\section{Discussion}

In this study, we assessed the safety, validity and functionality of an innovative rehabilitation equipment produced by Alreh Medical, the iStander, and its associated software, Neuroforma, as well as of a commercially available sensor produced by Fitbit, the Fitbit Charge 2. Patients hospitalized roforma only. Exercises were performed under monitoring by the Fitbit Charge 2. No major safety issues regarding devices used were reported by care-givers or patients. The functionality assessment of the iStander by the NTLI suggest that its global performance should be improved and the frustration associated to its use, decreased. More importantly, several suggestions of improvements were made by care-givers and patients and a more targeted use-case for the iStander was suggested (upper-limb training with trunk stabilization). Finally, a comparison of heart rate values measured by the Fitbit device and heart rate values measured by care-givers raised concerns about the potential use of the Fitbit Charge 2 as a heart rate measurement tool in the REACH context.

Our study has several limitations that are important to highlight. First of all, very small sample sizes were used and, accordingly, quantitative results (NTLI scale comparisons and HR comparison) may be affected by random error and could not be adjusted for potential confounder. A good illustration of this random error is that the characteristics of the randomly selected participants in each group are quite different. Reporting bias using the NTLI questionnaire is another potential issue as patients and care-givers were not blinded to the intervention. Accordingly, careful, preliminary conclusions only were drawn from quantitative results and we did not perform inference statistics. On the other hand, we believe that the use of qualitative methods such as free reporting of safety issues and free commenting on device functionality allowed us to collect very useful information for the improvement of the devices. Another concern is about the external validity of our study. Indeed, our inclusion criteria were the one defined by the REACH project for the Geneva use-case. These inclusion criteria describe rather fit patients of the geriatric division. Accordingly, results may be quite different in other populations, such as fit older adult living home or, on the other hand, more seriously impaired hospital patients. Altogether, this study was exploratory by nature and provide insights for the design of more structured trials with proper sample size, such as randomised-controlled trials. 
Heart Rate (HR) monitoring is a popular, versatile, convenient and non-invasive method that is readily used in a number of studies and tests ${ }^{8,9}$ for planning and monitoring the intensity of the physical activity ${ }^{10,11}$. Therefore, accuracy of its measurement is important. Some researchers propose that more frequent measurements and more accurate observation of HR would allow early detection of diseases or complications and prevent costly hospitalization, especially in the intensive care unit ${ }^{12-18}$. There are a number of scientific reports evaluating the accuracy of HR measurements using a wearable device compared to standard measurements. In the studies of Wallen et al., HR measurement using the Fitbit Charge HR was tested during activities such as sitting, lying, walking, running on a treadmill or pedaling on a cycloergometer ${ }^{19}$. The device underestimated the results, with a mean difference of a range 9.3-8.5 bpm. Dooley et al. observed that the Fitbit Charge HR measures much lower resting heart rate compared to standard measurements. Significant higher pulse readings were observed during light exercises and lower heart rate readings during intensive exercises ${ }^{20}$. Altogether, our results are concordant with the literature results and raise concerns about the validity of heart rate measurements by Fitbit devices. This would warrant further investigations with a dedicated study.

Importantly, no serious adverse events during the use of the devices were reported. Even people with cognitive or physical impairment were able to safely use the device. However, devices were used under the assistance of an occupational therapist. As a further step, the security of the devices with patients manipulating the devices alone should be assessed.

The study suggests that iStander in its current form would mainly be useful in neurorehabilitation for patients affected by severe limitations in lower limbs function. Indeed, the efficient trunk stabilization provided by the iStander would allow effective training for upper limbs but also for severely paretic lower limbs. Furthermore, the iStander was reported as well suited for squats but not so good for other lower limb exercises because of its fixed base of support. Occupational therapists reported the need of wider functionality than just verticalisation. Accordingly, the development of the iStander during the REACH project should be directed towards allowing comprehensive training of muscles involved in the walking process which is one of the main determinants of a senior's independence. The device should allow to safely carry out exercises to strengthen muscles of back, abdomen, legs and buttocks as well as to increase balance and prevent falls.

In our study, a low interest towards the videogame to stimulate rehabilitation was reported among some participants. Improvements are needed to engage the person deeper, especially for the interface. Motivation for regular exercise may come from a number of sources such as strengthening of a sense of self-reliance and independence, attractiveness of the human-computer interface or fear of progressive dementia ${ }^{21}$.

Monitoring the daily activity, heart rate or other health parameters of the elderly may allow to early detect a change in the health status and to respond accordingly in a targeted manner, for example by stabilizing the patient before a fall occurs or by proposing a targeted motivation strategy to a sedentary patient. Accordingly, monitoring, early detection and targeted intervention should be an important part of the mobility device, as described in the general concept of the REACH project.

Cognitive impairment and physical inactivity are quite linked. For example, dementia is positively correlated with decreased physical activity, fear of falling and associated pain ${ }^{22}$. Erickson et al. showed that regular physical exercises effectively reverse hippocampal volume loss which is connected to improved memory function ${ }^{23}$. Accordingly, the personal mobility device which will be created in touchpoint cluster 1 should tackle both issues. Supporting this statement, numerous evidences suggest a high efficiency of a combination of cerebral and physical training. For example, training of cognitive function in combination with task oriented physical activity can reduce the risk of falls among older adults. Moreover, dual-tasking exercises are considered as a critical health care need for improving balance and gait ${ }^{24}$.

In conclusion, no major safety issues with the iStander, its associated software, Neuroforma, and the Fitbit Charge 2 were reported in our study. Important comments about the functionality of the iStander were collected and requirements for its possible evolution towards the personal mobility device of touchpoint cluster 1 were discussed. Finally, uncertainties about the validity of heart rate measurements by the Fitbit devices were raised. Altogether, our study, even if exploratory, provides important insights for the continuation of the REACH project and, in particular, for the development of the touchpoint cluster 1 personal mobility device.

\section{Acknowledgments}

We sincerely thank the physical therapy and occupational therapy teams at the Hôpital des Trois-Chênes for their active and enthusiastic participation in the study.

\section{References}

1. The state of ageing and health in Europe. Merc \& Co., Inc, Whitehouse Station USA: International Longevity Centre-UK and The Merc Company Foundation, 2006 
2. Pitsavos C, Kavouras SA, Panagiotakos DB, Pitsavos C, Kavouras SA, Panagiotakos DB, Arapi S, Anastasiou CA, Zombolos S, Stravopodis P, Mantas Y, Kogias Y, Antonoulas A, Stefanadis C. Physical activity status and acute coronary syndromes survival. Journal of the American College of Cardiology 2008;51:2034-2039; https://doi. org/10.1016/j.jacc.2008.01.053

3. National Center for Chronic Disease Prevention and Health Promotion. At a glance-Healthy aging: Preventing disease and improving quality of life among older Americans 2004. Centers for Disease Control and Prevention, Atlanta, GA; http://www.cdc.gov/nccdphp/aag/aag_aging.htm; retrieved: May 20, 2017

4. Evans WJ. Exercise training guidelines for the elderly. Medicine and Science in Sports Exercise 1999;31(1):12-17; https://doi.org/10.1097/00005768-199901000-00004

5. Leveille SG, Guralnik JM, Ferrucci L, Langlois JA. Aging successfully until death in old age: opportunities for increasing active life expectancy. American Journal of Epidemiology 1999;149(7):654-664; https://doi.org/10.1093/oxfordjournals.aje.a009866

6. Psaltopoulou T, Kyrozis A, Stathopoulos P, Trichopoulos D, Vassilopoulos D, Trichopoulou A. Diet, physical activity and cognitive impairment among elders: the EPIC-Greece cohort (European Prospective Investigation into Cancer and Nutrition). Public Health Nutrition 2008;11:10541062; https:// doi.org/10.1017/s1368980007001607

7. Nazari G, MacDermid JC, Kin KE, Richardson J, Tang A. Reliability of Zephyr Bioharness and Fitbit Charge Measures of Heart Rate and Activity at Rest, During the Modified Canadian Aerobic Fitness Test and Recovery. The Journal of Strength \& Conditioning Research 2017, https:// doi.org/10.1519/JSC.0000000000001842

8. Mann T, Lamberts RP, Lambert MI. Methods of prescribing relative exercise intensity: physiological and practical considerations. Sports Medicine 2013;43(7):613-625; https://doi.org/10.1007/s40279-013-0045-x

9. Sirard JR, Pate RR. Physical activity assessment in children and adolescents. Sports Medicine 2001;31(6):439-454; https://doi.org/10.2165/00007256-200131060-00004

10. Hills AP, Mokhtar N, Byrne NM. Assessment of Physical Activity and Energy Expenditure: An Overview of Objective Measures. Frontiers in Nutrition 2014;1:5; https://doi.org/10.3389/fnut.2014.00005

11. Stahl SE, An HS, Dinkel DM, Noble JM, Lee JM How accurate are the wrist-based heart rate monitors during walking and running activities? Are they accurate enough? BMJ Open Sport and Exercise Medicine 2016;2(1):e000106; https://doi. org/10.1136/bmjsem-2015-000106

12. Schutz $Y$, Weinsier RL, Hunter GR. Assessment of freeliving physical activity in humans: an overview of currently available and proposed new measures. Obesity 2001;9(6):368-79; https://doi.org/10.1038/oby.2001.48

13. Kroll RR, Boyd JG, Maslove DM. Accuracy of a WristWorn Wearable Device for Monitoring Heart Rates in Hospital Inpatients: A Prospective Observational Study. Eysenbach G, editor. Journal of Medical Internet Research 2016;18(9):e253; https://doi.org/10.2196/jmir.6025

14. Smith ME, Chiovaro JC, O'Neil M, Kansagara D,
Quiñones AR, Freeman M, Motu'apuaka ML, Slatore CG. Early warning system scores for clinical deterioration in hospitalized patients: a systematic review. Annals of the American Thoracic Society 2014;11(9):145465; https://doi.org/10.1513/ AnnalsATS.201403-102OC

15. Churpek MM, Yuen TC, Park SY, Meltzer DO, Hall JB, Edelson DP. Derivation of a cardiac arrest prediction model using ward vital signs. Critical care medicine 2012;40(7):2102-2108; https://doi. org/10.1097/CCM.0b013e318250aa5a

16. Churpek MM, Yuen TC, Winslow C, Meltzer DO, Kattan MW, Edelson DP. Multicenter Comparison of Machine Learning Methods and Conventional Regression for Predicting Clinical Deterioration on the Wards. Critical Care Medicine 2016;44(2):368-74; https://doi.org/10.1097/ CCM.0000000000001571

17. Churpek MM, Yuen TC, Winslow C, Hall J, Edelson DP. Differences in vital signs between elderly and nonelderly patients prior to ward cardiac arrest. Critical Care Medicine 2015;43(4):816-22; https://doi.org/10.1097/CCM.0000000000000818

18. Churpek MM, Yuen TC, Park SY, Gibbons R, Edelson DP. Using electronic health record data to develop and validate a prediction model for adverse outcomes in the wards. Critical Care Medicine 2014;42(4):841-848; https://doi.org/10.1097/ CCM.0000000000000038

19. Wallen MP, Gomersall SR, Keating SE, WisløffU, Coombes JS. Accuracy of Heart Rate Watches: Implications for Weight Management. PLoS ONE 2016;11(5): e0154420; https://doi.org/10.1371/journal.pone.0154420

20. Dooley EE, Golaszewski NM, Bartholomew JB. Estimating Accuracy at Exercise Intensities: A Comparative Study of Self-Monitoring Heart Rate and Physical Activity Wearable Devices. Keepanasseril A, editor. JMIR mHealth and uHealth. 2017;5(3):e34; https://doi.org/10.2196/mhealth.7043

21. Mehra S, Dadema T, Kröse BJA, Visser B, Engelbert RHH, Van Den Helder J and Weijs PJM. Attitudes of Older Adults in a Group-Based Exercise Program Toward a Blended Intervention; A FocusGroup Study. Frontiers in psychology 2017;7:1827; https://doi.org/10.3389/fpsyg.2016.01827

22. Bherer L, Erickson KI, Liu-Ambrose T. A Review of the Effects of Physical Activity and Exercise on Cognitive and Brain Functions in Older Adults. Journal of Aging Research 2013;2013:657508; https://doi.org/10.1155/2013/657508

23. Erickson KI, Voss MW, Prakash RS, Basak C, Szabo A, Chaddock L. Exercise training increases size of hippocampus and improves memory. Proceedings of the National Academy of Sciences of the United States of America. 2011;108(7):3017-3022; https://doi.org/10.1073/pnas.1015950108

24. Silsupadol P, Shumway-Cook A, Lugade V, van Donkelaar P, Chou L-S, Mayr U. Effects of Single-Task Versus Dual-Task Training on Balance Performance in Older Adults: A Double-Blind, Randomized Controlled Trial. Archives of physical medicine and rehabilitation 2009;90(3):381-387; https://doi.org/10.1016/j.apmr.2008.09.559 